\title{
Reducing or Cancellations and Hospital Cost: Nurse -Led Intervention at a Tertiary Care Hospital in LMIC
}

\author{
Sadaqat Ali $^{1 *}$, Tahira faiz ${ }^{1}$, DR Waris Ahmed ${ }^{2}$, DR Mahim Malik Akmal ${ }^{2}$ \\ ${ }^{1}$ Nurse Practitioner, Congenital heart surgery, Aga Khan University Hospital, Pakistan \\ ${ }^{2}$ Assistant Professor, Cardiothoracic surgery, Aga Khan University Hospital, Pakistan
}

DOI: $\underline{10.36348 / \text { sjnhc.2021.v04i04.001 }}$

| Received: 04.03.2021 | Accepted: 03.04.2021 | Published: 07.04.2021

*Corresponding author: Sadaqat Ali

\section{Abstract}

Background: Surgical cancellations at the day of surgery present major difficulties to both health care organizations as well patients and families. In a low middle income country (LMIC), cancellations stress an already resource limited system and result in significant financial burden and loss of resources. Due to a limited number of tertiary care hospitals, most families have to travel great distances and outpatient visits for preoperative assessment are unfeasible. We describe a protocol using screening phone calls and patient's family counseling prior to admission to reduce same-day cancellations rates. Objective: To determine whether implementation of nurse -led protocol involving screening phone call and standardized questionnaire, along with patient's family counseling 48 hours prior to surgery results in reducing day-of-surgery cancellations. Methods and measures: Pre-intervention data to calculate rate of cancellations was collected. Patient's families were called 48 hours prior to surgery. A standard assessment checklist was used to cover pertinent points. A brief counseling session was also carried out regarding the surgery .In case of any concerns, attending surgeon and cardiologist were informed and a follow up call made on the day before surgery. A satisfaction survey regarding the effectiveness of the phone call was carried out after discharge from the hospital. Post intervention data was then collected and cancellation rate calculated. Results: Post-intervention, data from 144 screened patients was collected. Day of surgery cancellation rate decreased from $15.2 \%$ to $1.43 \%(\mathrm{p}<0.05)$ (fig 3). An average cost of $\$ 540$ (Pakistan Rupee 80000) was saved per cancellation. Most families reported overall satisfaction with the phone call. Conclusion: Implementation of standardized protocol for preoperative assessment has decreased day of surgery cancellations, resulting in better resource utilization and reducing overall hospital costs. It has also helped in improving communication between patient's family and heath care providers and improved overall patient satisfaction.

Keywords: Hospital Cost, Nurse -Led Intervention LMIC.

Copyright (C) 2021 The Author(s): This is an open-access article distributed under the terms of the Creative Commons Attribution 4.0 International License (CC BY-NC 4.0) which permits unrestricted use, distribution, and reproduction in any medium for non-commercial use provided the original author and source are credited.

\section{INTRODUCTION}

Day of surgery cancellations are a significant source of frustration and increased costs for both hospital systems as well as patients and their families. This issue carries even more significance in developing countries where health care resources are limited. The disparity between disease burden and centers equipped to dealing with increasingly complex problems leads to overburdening of an already over-taxed system. Cancellation of surgery becomes a major problem in these over stretched, resource limited setting, adding to the financial burden and reducing the unit's overall efficiency [1].

A study conducted at our own setting has shown that while patient related factors leading to cancellations were difficult to control, some factors such as administration and logistics related issues can be countered with better communication and organizational strategies [2]. With this project, we sought to evaluate whether patient compliance and overall efficiency could be increased by performing a pre-operative assessment via phone call 48 hours prior to surgery, along with developing feedback mechanisms, to decrease same day cancellation rate of surgeries.

\section{MATERIALS AND METHODS}

The project was initiated as a Quality Initiative led by advanced nurse practitioners (ANP) on the Congenital Cardiothoracic Surgery Service at a tertiary care hospital. We perform an average of 250-300 open heart cases a year. According to the existing protocol, patients are seen in outpatient clinic by the surgery 
Sadaqat Ali., Saudi J Nurs Health Care, Apr, 2021; 4(4): 91-98

service after initial pediatric cardiology evaluation. They are then admitted the night before surgery and undergo pre-operative anesthesia evaluation as inpatient. Since at least half of our patients travel significant distances to get to the hospital, we are unable to schedule a preoperative clinic visit. Surgical bookings are documented manually in a ledger maintained by the ANPs as well as in the online operating room electronic system.

Appropriate Institutional Review Board exemption was sought according to institutional guidelines. The project was divided into 3 phases. In the pre-intervention phase (extending from January to March 2018) data was collected prospectively on the number of patients admitted the night before surgery and then cancelled the next day. Reason for cancellation was recorded in a secure database, summarized and discussed in a multidisciplinary team (consisting of surgeons, pediatric cardiologists and ANPs). Key reasons for cancellations were identified and subsequently a protocol was developed. This consisted of a standardized questionnaire checklist (figure 1) which was administered over the phone to the patient's guardian 48 hours prior to surgery. This covered all pertinent information including: patient's current medical condition (specifically conditions that would preclude surgery such as fever, cold, rash), current medications, as well as logistics; in addition to any queries that they may have. In case of any concerns, attending surgeon and cardiologist were informed and a follow up call made on the day before surgery. The family was also provided additional information and counseling regarding the surgery. The protocol was implemented in April 2018, and data was collected prospectively for a period of 3 months after implementation of protocol till June 2018. During this time, a satisfaction survey (Figure 2) regarding the patient and family's perception about the protocol was also conducted after discharge from the hospital. Six months after implementation of the protocol, we performed an audit of the process to assess sustainability, from January 2019 to June, 2019 (Stage 3 , sustainability phase).

\section{Phone call checklist}

Patient Name:

MRN

Procedure

Respondent

Phone number

Date of Phone call

Questions

1- Does the child have any cold or flu symptoms YES

2. Does the child have diarrhea/vomiting

3. Does the child have fever.

4. Does the child have any rashes currently

5. Do you have blood arranged?

6. Have you made arrangements (financial, transport etc.) for surgery?

7. What medications is the child currently taking?

\section{Family counseling: \\ -Procedure \\ - Post operative expectations \\ Will this patient stay on schedule?}

$\begin{array}{ll}\text { YES } & \text { NO } \\ \text { YES } & \text { NO } \\ \text { YES } & \text { NO }\end{array}$

Fig-1: (Questioner) 
MRN:

Date of Surgery:

Date of Survey:

\begin{tabular}{|c|c|c|c|c|c|c|}
\hline & $\begin{array}{l}1 \\
\text { Strongly } \\
\text { Disagree }\end{array}$ & $\begin{array}{l}2 \\
\text { Disagree }\end{array}$ & $\begin{array}{l}3 \\
\text { Slightly } \\
\text { Disagree }\end{array}$ & $\begin{array}{l}4 \\
\text { Slightly } \\
\text { Agree }\end{array}$ & $\begin{array}{l}5 \\
\text { Agree }\end{array}$ & $\begin{array}{l}6 \\
\text { Strongly } \\
\text { Agree }\end{array}$ \\
\hline \multicolumn{7}{|l|}{$\begin{array}{l}\text { The preoperative assessment phone call was } \\
\text { helpful }\end{array}$} \\
\hline \multicolumn{7}{|l|}{$\begin{array}{l}\text { The preoperative assessment phone call } \\
\text { cleared doubts about surgery }\end{array}$} \\
\hline \multicolumn{7}{|l|}{ I felt reassured after the phone call } \\
\hline \multicolumn{7}{|l|}{$\begin{array}{l}\text { The health care provider provided } \\
\text { information to care for my child's health }\end{array}$} \\
\hline \multicolumn{7}{|c|}{$\begin{array}{l}\text { The health care provider gave me opportunity } \\
\text { to ask questions }\end{array}$} \\
\hline \multicolumn{7}{|l|}{$\begin{array}{l}\text { The preoperative assessment phone call } \\
\text { helped me prepare for the postoperative } \\
\text { period }\end{array}$} \\
\hline \multicolumn{7}{|l|}{ I am overall satisfied with the care delivered } \\
\hline $\begin{array}{l}\text { Additional preoperative clinic visit would } \\
\text { have been helpful }\end{array}$ & & & & & & \\
\hline
\end{tabular}

Additional comments:

Fig-2: Satisfaction Patient Satisfaction Survey for Pre-op assessment

\section{Result* Phase Cross tabulation Count}

\begin{tabular}{|l|c|c|c|c|c|}
\hline \multirow{2}{*}{} & \multicolumn{3}{|c|}{ Phase } & \multirow{2}{*}{ Total } & $\begin{array}{c}\text { Pearson chi- } \\
\text { square test }\end{array}$ \\
\cline { 2 - 5 } & Not Screened & Screened & Post Screened & & p-value \\
\hline Result Performed & 78 & 55 & 139 & 272 & 0.004 \\
Cancelled after admission & 14 & 4 & 5 & 23 & \\
Total & 92 & 59 & 144 & 295 & \\
\hline
\end{tabular}

Fig-3: Statistical Analysis

Chi-Square Tests

\begin{tabular}{|l|c|c|c|}
\hline & Value & df & $\begin{array}{c}\text { Asymp. Sig. } \\
\text { (2-Sided) }\end{array}$ \\
\hline Pearson chi-Square & $9.803^{\mathrm{a}}$ & 2 & .004 \\
Likelihood Ratio & 9.072 & 2 & .011 \\
Linear-by-Linear Association & 9.207 & 1 & .002 \\
N of Valid Cases & 315 & & \\
\hline
\end{tabular}

1 cell $(16.7 \%)$ has expected count less than 5 . The minimum expected count is 4.68.

\section{INCLUSION CRITERIA}

All patients undergoing elective congenital heart surgery at the Aga Khan University Hospital.

\section{EXCLUSION CRITERIA}

All emergent cases Patients already admitted for other medical issues.

\section{STATISTICAL ANALYSIS}

All analyses were carried out in SPSS version 21. Results are presented as mean and standard deviation for continuous variables, and frequency and percentages for categorical variables. Chi-Square tests used to see the mean difference between pre and post intervention and $\mathrm{p}$-value of $<0.05$ taken as significant.

\section{RESULTS}

In the first phase (pre-intervention) data was collected for a three month period, from January 2018 to March, 2018. A Total of 94 cases were scheduled for surgery, out of which $78(82.97 \%)$ cases were performed and $14(17.94 \%)$ cases were cancelled on the day of surgery (figure 4a). These included: 10 cancellations that were determined to be patient related issues such as viral illness and potentially preventable, 
Sadaqat Ali., Saudi J Nurs Health Care, Apr, 2021; 4(4): 91-98

2 cancellations were due to non-availability of space in ICU and were subsequently rescheduled, and another two were due to financial issues and family were unable to complete the financial arrangement .This was potentially preventable reason though this was pre intervention phase and the given data was collected from admission department record. After an initial washout period, the protocol was implemented, as detailed above from April, 2018 to June, 2018. (Figure 4b) Data was collected prospectively including the admissions, the number of surgeries that were performed as well as the cancellations. A satisfaction survey was also conducted during this time, in order to evaluate the overall perception in terms of family satisfaction. In this phase, a total of 87 cases were scheduled in which 59 were screened for surgery, 28/87 were not screened as they did not fulfill the inclusion criteria for the study and were thus excluded. 04 out of the $59(7.27 \%)$ cases were canceled prior to admission after screening, including two cases due to viral illness, and two due to family refusal for surgery. Only $2(3.63 \%)$ patients were cancelled after admission on day of surgery. One of which was canceled due to raised leucocytes counts, the second due to nonavailability of beds in PCICU.

In third phase which is Sustainability phase (figure 4c) from January 2019 to June, 2019, total 144 surgeries were planned in which 139 were screened for surgery. Out of 139 only 2(1.43\%) cases were canceled after admission due to financial issue and remaining $137(98.56 \%)$ cases were performed. During screening, 05 cases were cancelled and/or rescheduled after the screening phone call due to the following reasons: three were related to viral illnesses and two were due to logistic issues faced by the family. These OR slots were then allocated to other patients, thus avoid potential wastage of resources.

In conclusion, the percentage for case cancellation after admission decreased from $14.89 \%$ (14/94 patients) in the prescreening phase to $3.77 \%$ $(2 / 54, p<0.05)$ in the implementation phase. Moreover, in sustainability phase (Figure $4 \mathrm{~b}$ ) it was decreased to $1.43 \%(2 / 139, \mathrm{p}<0.05)$ (Figure $4 \mathrm{C})$.

Fig-4: $(a+b+c)$ the flow diagrams for each phase

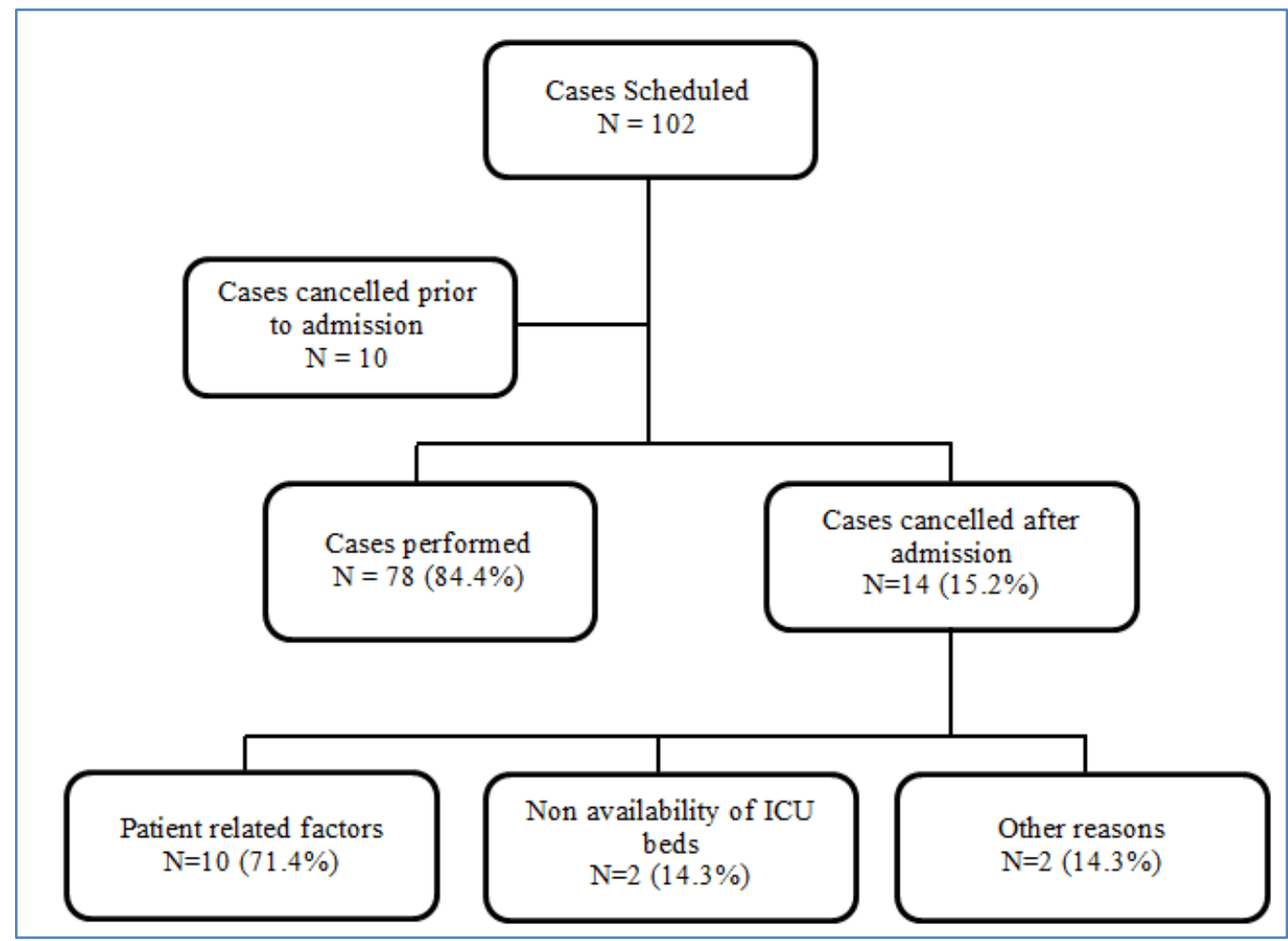

Fig-4(a): Pre Intervention Phase 
Sadaqat Ali., Saudi J Nurs Health Care, Apr, 2021; 4(4): 91-98

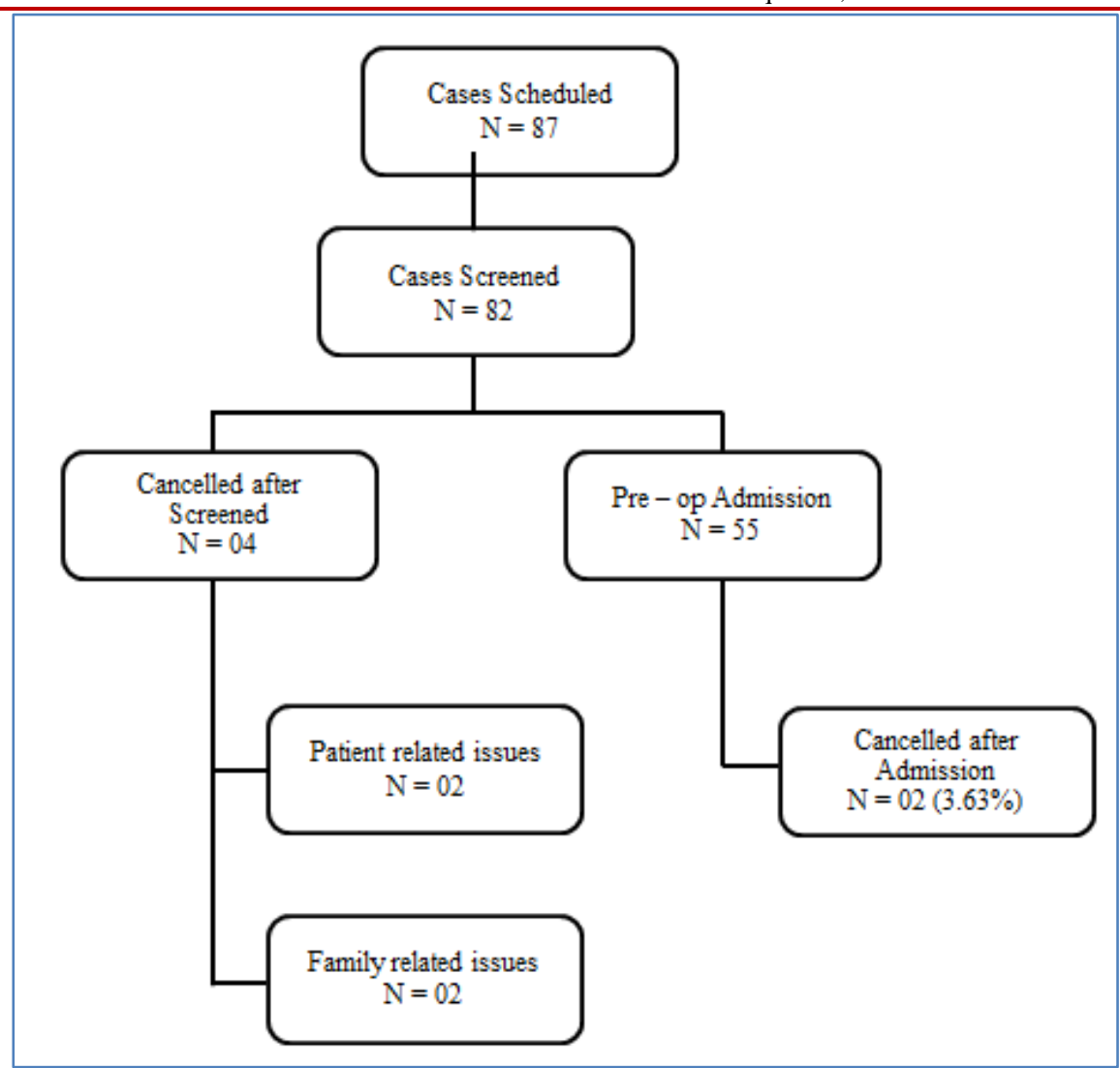

Fig-4(b): Implementation Phase

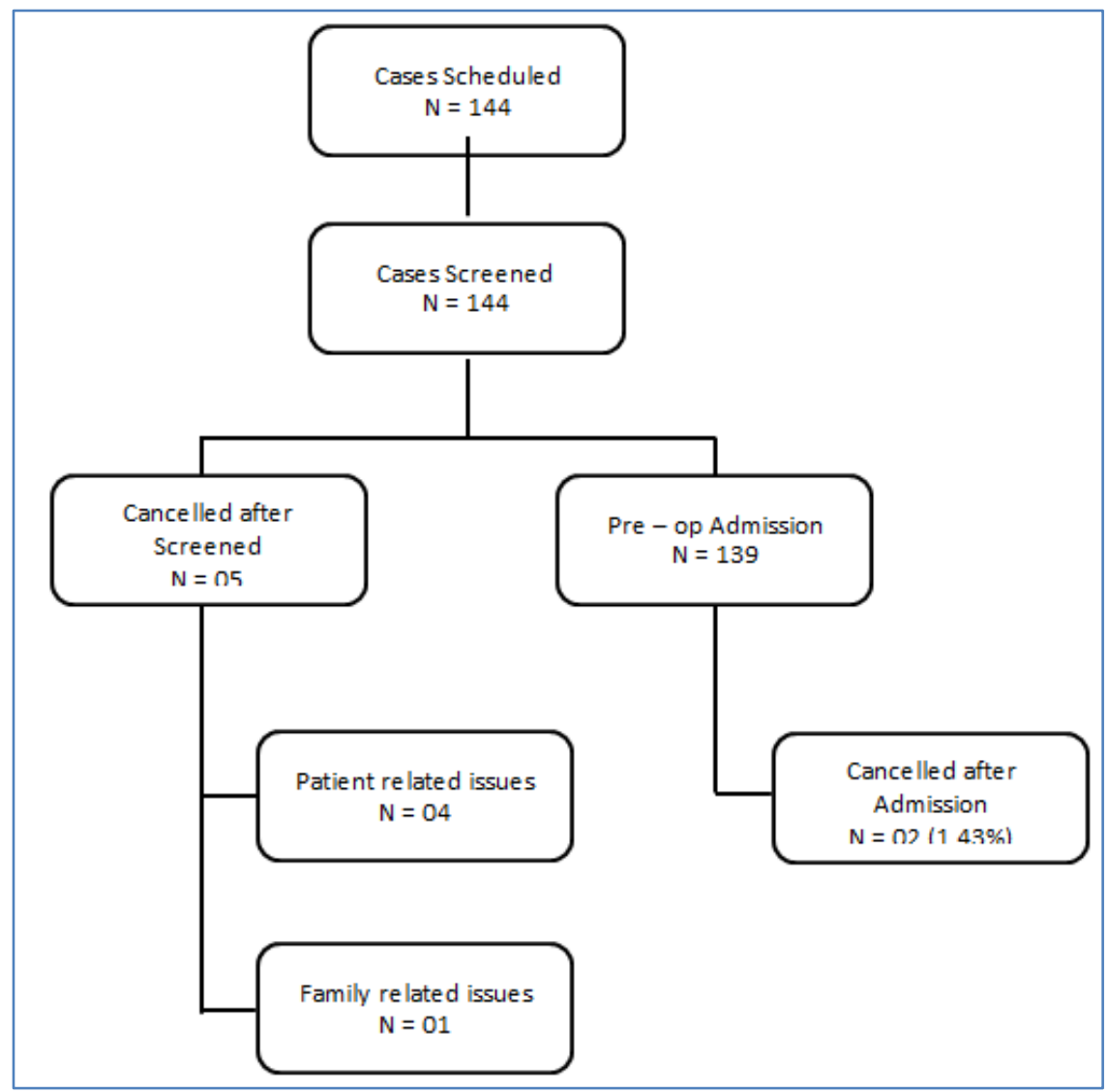

Fig-4(c): Sustainable Phase 
Sadaqat Ali., Saudi J Nurs Health Care, Apr, 2021; 4(4): 91-98

\section{Financial Costs}

On average, each preoperative admission costs a total of 13000 Rupees (bed charges PRs 5000 per day, preoperative lab work PRS 7000), in addition to Operating Room charges of PRs 40,000 per hour. So on approximately PRS 100,000 equal to 645 US dollars is lost per cancelled surgery.

\section{Satisfaction among patient's families}

Furthermore, satisfaction survey about the phone call was also conducted after surgery during both Implementation and sustainability phase. (Figure 5) In implementation phase, most of the caregivers strongly agreed that the call was very helpful for them and the preoperative assessment phone call had cleared their doubts about surgery and they felt reassured after the phone call. Although, few of them were less satisfied that the health care provider gave them opportunity to ask questions, and the preoperative assessment phone call helped them to prepare for the postoperative period. Overall, they were satisfied with the care delivered.
These results were discussed in our multidisciplinary quality meeting and led to re-evaluation of the counseling provided during the screening phone call. A second survey was conducted in the sustainability-phase which showed improvement in the overall satisfaction scores. Results from pre-screening phase in which all patient answers were in the categories of strongly agreed and agreed in response to all the questions, but in last question that is "additional preoperative clinic visit would have been helpful for them" some patients responses were falling in the category of strongly agreed. However, some agreed to that statement while few participants marked their responses under the category of slightly agreed and remaining participants disagreed to that question.

In conclusion implementation of phone call checklist significantly reduced surgery cancellation and most of the patients were satisfied with the information given via phone call.

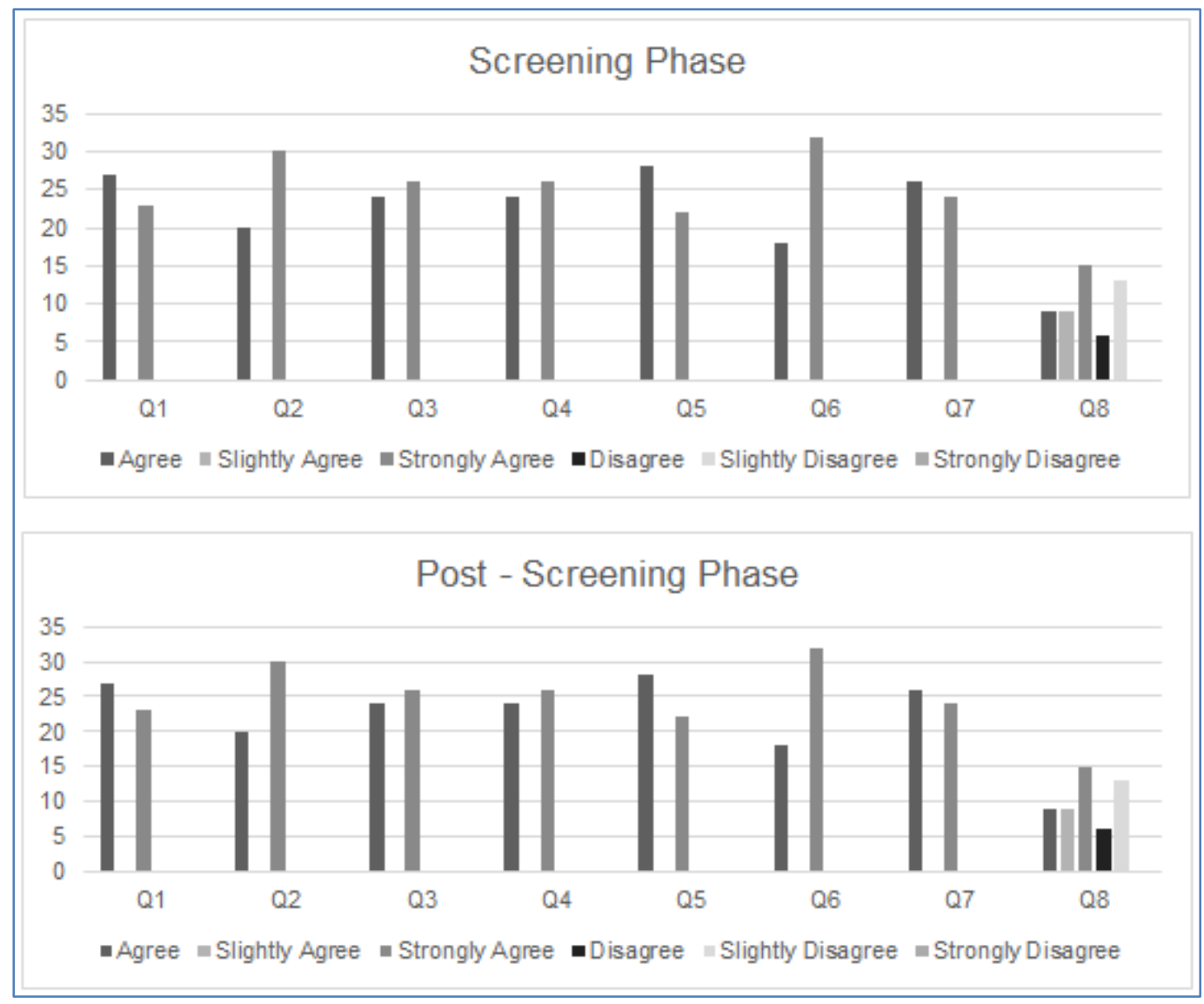

Fig-5: Satisfaction Analysis

\section{DISCUSSION}

In most health care settings, day care and inpatient surgeries remain the highest source of revenue. Therefore unprecedented case cancellations create significant anxiety in not just patients and families, but also health care providers and hospital administrators. In low middle income countries like
Pakistan, where both access and delivery of health care faces multiple challenges, case cancellations result in loss of revenue not only for the health care system, but also increases the financial burden on the patients and their families. Multiple studies have reinforced these observations that case cancellation is major reason in 
Sadaqat Ali., Saudi J Nurs Health Care, Apr, 2021; 4(4): 91-98

poor patient satisfaction and hospital patient relationship and training [3].

In a cash-for-service system, this potentially avoidable financial burden has far reaching implications. Studies done in the Past have extensively investigated reasons behind these cancellations and various methodologies to reduce [4]. While developing a process that best suited our own system, we felt that a screening phone call performed a certain time period prior to surgery would be the most cost effective way to screen potential cancellations. All stakeholders also felt that this would also allow the families direct contact with the surgical team, whom, in some cases, they had not met since the initial evaluation. Previous studies have shown the effectiveness of these methods although none have shown the cost implications [5]. Haufler and Harrington reported that preoperative nurses at a North Carolina ASC successfully decreased the daily rate of cancellation by $53 \%$, increased patient satisfaction scores from $89 \%$ to $94 \%$, and increased OR use from $72.4 \%$ to $75.8 \%$ by employing a nurse-to-patient phone call protocol [6]. Several studies have suggested that patient and guardian education and use of an effective tool in gathering important health information, performance of a complete preoperative assessment, and effective communication with patients and families all played a significant role in positive patient outcomes and prevention of surgical cancellations [7]. The maximum cause for case cancellation was diagnosis related, which comprised $25.8 \%$ of all cancellations, which was followed by coordination causes at $15.1 \%$.In regards, the most common cause of all is preoperative diagnosis including diagnosis, coordination about sudden detrition in Health status. In order to reduce such events, strategic planning is very crucial and the most important aspect in this regards is appropriate preoperative assessment and coordination with family [8]. The major reason for cancellations was "patient unfit for operation" $(27.7 \%)$, "resource-related reasons" $(24.2 \%)$ and "no need for operation" $(23.5 \%)$.

There were 6(1\%) patients who were inadequately prepared pre-operative compared to $13 \%$ by Jonnalagadda et al. and $10 \%$ by Windokun et al. Jonnalagadda et al. also showed that $9 \%$ of the patients were absent or did not show up [9]. Similarly, in the study by Garg R et al., 16.2\% patients did not show up for their booked surgery [10]. In our study 3(0.5\%) patients refused consent on the operative day and in $1(0.2 \%)$ case the patient did not show up for their elective surgery. Late refusal or patient no-show can be endorsed to nervousness, fear or personal issues. Virtuous and through counselling to the patients regarding the nature of their procedure and type of anesthesia involved will belay most of their fears. Lack of patient doctor communication can be indorsed to these cases [11]. Another study has shown that cost of surgery cancelation was considerably high in the Shohada Kargar hospital. More than half of the cost
$(62.1 \%)$ of surgery cancelation was due to avoidable reasons. To avoid cancellations, we recommend performing preoperative visits with all patients [12]. In a university of Finland, a study was conducted to assess the cost of case cancellation, According to the results, a financial loss of nearly a million euros occur during the nine-month study period. By specialty, the cost of a single day of surgery cancellation was highest in cardiac and thoracic and vascular surgery and lowest in ophthalmology. By reason for cancellation, a major loss was related to hospital resources and patients' suitability for the planned surgery [13]. A previous study conducted in the department of general surgery at Aga Khan University hospital showed that average patient related factors were uncontrollable but some of them like anesthesia related cancellation are modifiable by better communication between surgeon and the administrative team, while logistic issues can be reduced with better organizational strategies [14].

According to the results of a recent census in Pakistan, the median income of an average family in Pakistani Rupees is 18000 to 25000 (US\$140 to US\$ 200) per month [15]. In that regard, a cost reduction of $540 \$$ per case is a significant cost for moth the hospital system as well the patients and their families.

Certainly, our study has major limitations. The foremost was the implementation of such a quality initiative project in our setting. Currently there is no existing framework for outpatient patient communication or home health care delivery. We do not have the resources to dedicate a team to this service. Consequently, during the early period of implementation, we had an almost $15 \%$ rate of undetection, mostly due to non-availability of staff for this task. As we developed during the course of the project and the processes became more streamlined, we were able to effectively screen all our preoperative patients. Secondly, another major limitation was the communication with the patient's families. Pakistan has a diverse cultural background, with over 80 regional languages spoken. We, on occasion, felt that due to language barriers we were unable to provide the appropriate counseling.

\section{CONCLUSION}

Over all Implementation of standardized protocol for preoperative assessment has significantly decreased day of surgery cancellations, resulting in better resource utilization and reducing overall hospital costs. It has also helped in improving communication between patient's family and heath care providers and improved overall patient satisfaction.

\section{REFERENCES}

1. Cho, H. S., Lee, Y. S., Lee, S. G., Kim, J. M., \& Kim, T. H. (2019). Reasons for surgery cancellation in a general Hospital: a 10-year 
Sadaqat Ali., Saudi J Nurs Health Care, Apr, 2021; 4(4): 91-98

study. International journal of environmental research and public health, 16(1), 7.

2. Monem, A., Nadeem, A., Khan, F.A. Audit Article.

3. Sultan, N., Rashid, A., \& Abbas, S. M. (2012). Reasons for cancellation of elective cardiac surgery at Prince Sultan Cardiac Centre, Saudi Arabia. Journal of the Saudi Heart Association, 24(1), 29-34.

4. Dimitriadis, P. A., Iyer, S., \& Evgeniou, E. (2013). The challenge of cancellations on the day of surgery. International Journal of Surgery, 11(10), 1126-1130.

5. Kumar, R., \& Gandhi, R. (2012). Reasons for cancellation of operation on the day of intended surgery in a multidisciplinary 500 bedded hospital. Journal of anaesthesiology, clinical pharmacology, 28(1), 66.

6. Haufler, K., \& Harrington, M. (2011). Using nurse- to- patient telephone calls to reduce dayof- surgery cancellations. AORN journal, 94(1), 19-26.

7. Dye, J., \& Kennedy, R. (2008). Peri-operative Patient Education: What Methods of Patients Education Will Better Prepare Patients for Their Surgical Experience; and Will Better Preparation Result in Increased Patient Satisfaction (Doctoral dissertation).

8. Yu, K., Xie, X., Luo, L., \& Gong, R. (2017). Contributing factors of elective surgical case cancellation: a retrospective cross-sectional study at a single-site hospital. BMC surgery, 17(1), 1-8.

9. Shiraz, D., Naeem, T., Rafiq, M. S., \& Khan, M. M. (2016). Cancelled and postponed elective cases in general surgery. Pak J Surg, 32(3), 162-167.

10. Garg, R., Bhalotra, A. R., Bhadoria, P., Gupta, N., \& Anand, R. (2009). Reasons for cancellation of cases on the day of surgery-a prospective study. Indian journal of anaesthesia, 53(1), 35.

11. Raju, B., \& Reddy, K. (2017). Are counseling services necessary for the surgical patients and their family members during hospitalization?. Journal of neurosciences in rural practice, 8(1), 114.

12. Maimaiti, N., Rahimi, A., \& Aghaie, L. A. (2016). Economic impact of surgery cancellation in a general hospital, Iran. Ethiopian Journal of Health Development, 30(2), 94-98.

13. Turunen, E., Miettinen, M., Setälä, L., \& Vehviläinen-Julkunen, K. (2018). Financial cost of elective day of surgery cancellations.

14. Ahmed, T., Khan, M., \& Khan, F. (2009). Cancellation of surgery in patients attending the preoperative anaesthesia assessment clinic: a prospective audit. Journal of the Pakistan Medical Association, 59(8), 547.

15. https://www.ilo.org/wcmsp5/groups/public/---asia/--ro-bangkok/---iloislamabad/documents/publication/wcms_554791.p df. 